Note: This is a preprint of paper being submitted for publication. Contents of this paper should not be quoted nor referred to without permission ot the author(s).

Submitted to the 1995 Materials Research Society Meeting, Boston, MA

\title{
Effect of Ambient Gas Pressure on Pulsed Laser Ablation Plume Dynamics and ZnTeFilm Growth
}

C. M. Rouleau, D. H. Lowndes, M. A. Strauss, ${ }^{1}$ S. Cao, ${ }^{*}$ A. J. Pedraza,* D. B. Geohegan, A. A. Puretzky,** and L. F. Allard

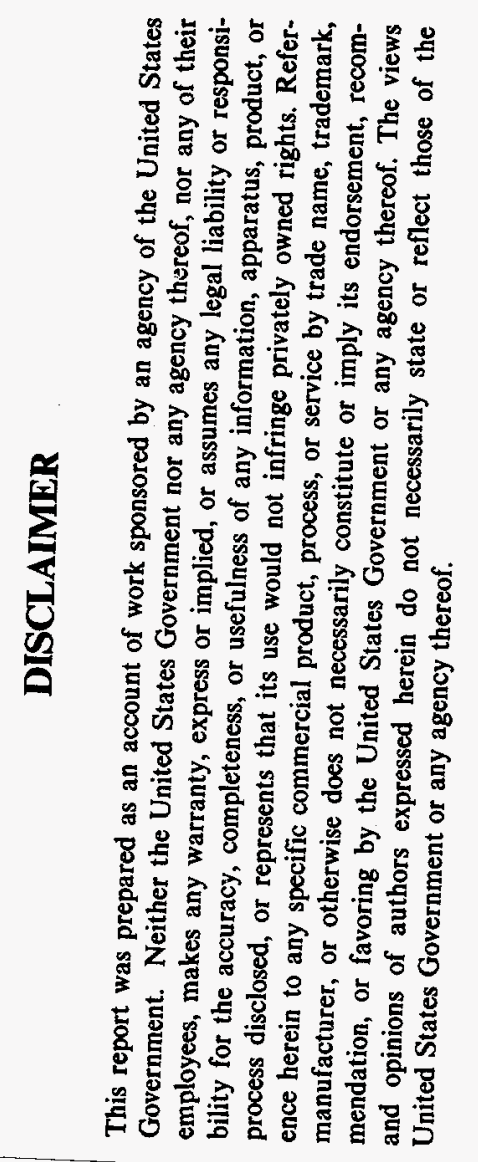

"The submitted manuscript has been authored
by a contractor of the U.S. Government under
contract No. DE-AC05-84OR21400,
Accordingly, the U.S. Government retains a
nonexclusive, royalty-free license to publish or
reproduce the published form of this
contribution, or allow others to do so, for U.S.
Government purposes."

December 1995

1 Dept. of Materials Science and Engineering

*The University of Tennessee, Knoxville, TN

**Institute of Spectroscopy, Troitsk, Russia

Prepared by

Solid State Division

Oak Ridge National Laboratory

P.O. Box 2008

Oak Ridge, Tennessee 37831-6056

managed by

LOCKHEED MARTIN ENERGY SYSTEMS, INC.

for the

U.S. DEPARTMENT OF ENERGY

under contract DE-AC05-84OR21400 


\section{EFFECT OF AMBIENT GAS PRESSURE ON PULSED LASER ABLATION PLUME DYNAMICS AND ZnTe FILM GROWTH}

C.M. ROULEAU, ${ }^{1}$ D.H. LOWNDES, ${ }^{1}$ M.A. STRAUSS, ${ }^{2}$ S. CAO, ${ }^{2}$ A.J. PEDRAZA, ${ }^{2}$ D.B. GEOHEGAN, ${ }^{1}$ A.A. PURETZKY ${ }^{4}$ and L.F. ALLARD ${ }^{3}$

${ }^{1}$ Solid State Division, Oak Ridge National Laboratory, Oak Ridge, TN 37831

${ }^{2}$ Dept. of Materials Science and Engineering, University of Tennessee, Knoxville, TN 37992.

${ }^{3}$ High Temperature Materials Laboratory, Oak Ridge National Laboratory, Oak Ridge, TN 37831

${ }^{4}$ Institute of Spectroscopy, Troitsk, Russia

\section{ABSTRACT}

Epitaxial thin films of nitrogen-doped p-ZnTe were grown on single-crystal, semi-insulating GaAs substrates via pulsed laser ablation of a stoichiometric ZnTe target. Both low pressure nitrogen ambients and high vacuum were used. Results of in situ reflection high energy electron diffraction (RHEED) and time-resolved ion probe measurements have been compared with ex situ Hall effect and transmission electron microscopy (TEM) measurements. A strong correlation was observed between the nature of the film's surface during growth (2-D vs. 3-D, assessed via RHEED) and the ambient gas pressures employed during deposition. The extended defect content (assessed via cross-sectional TEM) in the region $>150 \mathrm{~nm}$ from the film/substrate interface was found to increase with the ambient gas pressure during deposition, which could not be explained by lattice mismatch alone. At sufficiently high pressures, misoriented, columnar grains developed which were not only consistent with the RHEED observations but also were correlated with a marked decrease in Hall mobility and a slight decrease in hole concentration. Ion probe measurements, which monitored the attenuation and slowing of the ion current arriving at the substrate surface, indicated that for increasing nitrogen pressure the fast (vacuum) velocitydistribution splits into a distinct fast and two collisionally-slowed components or modes. Gascontrolled variations in these components mirrored trends in electrical properties and microstructural measurements.

\section{INTRODUCTION}

In little less than a decade, the field of pulsed laser ablation (PLA) has enjoyed rapid growth in both fundamental studies of the process itself as well as an increasing number of applications for deposition of thin films. For example, the technique has been successfully employed to grow a wide variety materials including complex oxides[1-5], II-VI compound semiconductors[6-12], IIII-VI chalcopyrites[13-20], and recently even column-III nitrides[21, 22]. Without exception, the quality of such films has been shown to be very sensitive to experimental parameters such as laser energy density, substrate temperature, target-to-substrate separation, and ambient gas pressure in cases where ambients are employed. The high- $T_{C}$ superconductor community, for instance, has long been aware that the pressure of oxygen employed during pulsed laser deposition greatly affects the electrical and structural properties of high- $\mathrm{T}_{\mathrm{c}}$ films $[1-5]$. Kim and $\mathrm{Kwok}$, for example, presented a scaling law for YBCO growth which relates oxygen pressure to target-to-substrate separation and have shown that specific combinations are required to grow optimum material[3]. Recently, a similar trend has been reported for II-VI compound semiconductors[9], suggesting that pulsed laser deposition in an ambient gas is beneficial to some extent. Although it is not clear that having gas molecules present on the growing film surface is beneficial to film quality, it is plausible that collisions with ambient gas molecules reduce the ablation beam's kinetic energy below the threshold for lattice displacement damage and point defect generation and thus films of higher quality should result.

The research reported here is part of a larger program to explore the use of pulsed laser ablation (PLA) to grow complex compound semiconductors [23]. PLA's ability to deposit films in ambient gases offers intriguing possibilities to control both the composition and doping of semiconductors $[10,11,21,22]$.

The purpose of this work was to obtain a more complete understanding of effects of ambient gases on compound semiconductor growth by using PLA of a stoichiometric ZnTe target through low pressure nitrogen ambients and high vacuum and correlating observed pressure-related-trends 
in RHEED observations and electrical properties with those of microstructural evolution and ablation plume dynamics.

\section{EXPERIMENTAL}

\section{Growth of ZnTe}

Film growth was carried out in a high vacuum $\left(\sim 10^{-7}\right.$ torr base pressure) pulsed laser deposition (PLD) system equipped with a differentially pumped reflection high energy electron diffraction (RHEED) system for assessing initial substrate surface cleaning and quality as well as subsequent epilayer quality. A pulsed $\mathrm{KrF}(248 \mathrm{~nm})$ excimer laser was used for ablation.

Cylindrical lenses were employed to bring the laser beam to a horizontal line focus along the radius of a rotating $(\sim 8 \mathrm{rpm})$ stoichiometric $\mathrm{ZnTe}$ target. The geometry of the growth chamber fixed the angle of incidence of the laser beam to $25^{\circ}$. The laser was operated so as to maintain a constant energy density at the target of approximately $0.7 \mathrm{~J} / \mathrm{cm}^{2}$. The 1 " diameter $\mathrm{ZnTe}$ target disk was commercially fabricated via hot isostatic pressing of high purity prereacted $\mathrm{Zn}$ and $\mathrm{Te}$ powders. In an effort to uniformly erode the target surface and prevent extensive cone formation, the ablation beam was periodically switched, in synchronization with the pulsing laser, from one half of the target to the other half throughout the growth.

$\mathrm{ZnTe}$ films were deposited on substrates cleaved from semi-insulating, epi-ready GaAs wafers that were miscut $2^{\circ}$ off [001] towards [110]. No wet chemical processes were employed before In bonding the substrate to the heater face. Before deposition, the substrates were preheated in high vacuum at $580^{\circ} \mathrm{C}$ for several minutes until a streaky RHEED pattern evolved. Growth commenced after cooling the substrate to $320^{\circ} \mathrm{C}$ and moving the heater into the growth position, $10 \mathrm{~cm}$ from the face of the target. The substrate heater was rotated at nearly $9 \mathrm{rpm}$ which, combined with the ablation beam switching, produced films with nearly uniform thickness. Film thickness was monitored in situ by employing a HeNe laser-based reflectance interferometer during deposition. All the films in this work were grown to a thickness in the range 0.5 to $0.7 \mu \mathrm{m}$ at a constant growth rate of nearly $1 \AA / \mathrm{s}$. The pulse-normalized deposition rate was in the range 0.1 to $0.5 \AA /$ pulse.

When deposition in an ambient was desired, a mass-flow controller was used to introduce ultra-high-purity gas ( $\mathrm{N}_{2}$ for these experiments) into the growth chamber through a port upstream from the pump. The total chamber pressure was controlled by a capacitance manometer and a downstream throttle valve. Vacuum, as well as $\mathrm{N}_{2}$ pressures of 25,50 and 100 mtorr (fixed flow rate of $4 \mathrm{sccm}$ ) were explored in this work.

\section{ZnTe Ablation Plume Diagnostics}

The interaction of the ablation plume with $\mathrm{N}_{2}$ was studied in a separate high vacuum $\left(\sim 10^{-6}\right.$ torr base pressure) chamber with adjustable gas flow rate and chamber pressure. The system also was equipped with a gated, intensified-CCD, lens-coupled camera system and a tunable liquid crystal filter for time- and spectrally-resolved imaging of the ablation plume, and an ion probe for charged particle measurements at various distances from the face of the ablation target. Optical emission from the plume was monitored using a spectrometer consisting of a $1.33 \mathrm{~m}$ monochromator equipped with a 1800 groove $/ \mathrm{mm}$ grating, a gated intensified diode array and a photomultiplier tube. When optical absorption measurements were required, the emission from a broadband xenon flash lamp was made to pass through the plume and into the spectrometer. A pulsed $\mathrm{KrF}$ excimer laser (the same model as was used for growth) was used for ablation.

Diagnostics were carried out using the target from the growth experiments and under nearly identical conditions, namely an energy density at the target of $\sim 0.7 \mathrm{~J} / \mathrm{cm}^{2}$ and a target-to-substrate (unheated) separation of $10 \mathrm{~cm}$. A number of $\mathrm{N}_{2}$ pressures were explored including those used in the growth experiments.

\section{RESULTS AND DISCUSSION}

RHEED pattern observations were made in two azimuths before and after every film deposition. In general, the substrate pattern before growth (after oxide desorption) was the same 
in all cases, a streaky (2D), high contrast pattern. However, as the pressure of either $\mathrm{N}_{2}$ or Ar was increased, there was a marked departure of the post-growth RHEED pattern away from the typical streaky pattern toward patterns having other characteristics. These observations are summarized in Table 1.

Table 1. RHEED pattern observations before and after film growth. Pattern observations for films grown in an ambient gas were performed after evacuating the chamber to high vacuum.

\begin{tabular}{|l|l|}
\hline \multicolumn{1}{|c|}{ Observation Condition } & RHEED pattern quality in 110 and 110 substrate directions \\
\hline Substrate surface before growth & Sharp, streaky, high contrast pattern \\
\hline Vacuum deposited film & Sharp, streaky, high contrast pattern \\
\hline 25 mtorr $\mathrm{N}_{2}$ deposited film & $\begin{array}{l}\text { Sharp, streaky, high contrast pattern having intensity along } \\
\text { streaks slightly modulated }\end{array}$ \\
\hline 50 mtorr $\mathrm{N}_{2}$ deposited film & Sharp, spotty, high contrast fish-net style pattern \\
\hline 100 mtorr $\mathrm{N}_{2}$ deposited film & More diffuse, spotty, lower contrast pattern* \\
\hline
\end{tabular}

* multiple diffractions were observed during azimuthal rotation.

The changes in the RHEED pattern as function of $\mathrm{N}_{2}$ pressure suggest a gradual departure from a smooth, highly ordered single crystal surface (streaky pattern) to one that is more threedimensional and less ordered (spotty pattern). The key difference between the diffraction pattern obtained from the 100 mtorr sample and those of the rest was the presence of multiple diffractions during azimuthal rotation which suggested surface features that were misoriented.

Hall effect measurements were employed at room temperature and at $77 \mathrm{~K}$ to determine the free hole concentration, $p$, and the free hole mobility, $\mu_{p}$ in the ZnTe:N films as a function of $N_{2}$ pressure in the growth chamber. Figure 1 shows measurements of $p$ and $\mu_{p}$ for $N_{2}$ pressures of 25,50 and 100 mtorr. The hole concentrations are nearly the same at room temperature and $77 \mathrm{~K}$ indicating heavy doping and the formation of an impurity conduction band. The figure also shows that both $\mu_{p}$ and $p$ peak in the vicinity of 50 mtorr and then each fall off to either side, $\mu_{p}$ falling more rapidly. We attribute the maxima in these parameters primarily to effects of the ablation plume's kinetic energy, at lower $\mathrm{N}_{2}$ pressures, and to excessive $\mathrm{N}$ incorporation and/or growth mode changes at higher $\mathrm{N}_{2}$ pressures. (High resolution $\mathrm{x}$-ray diffraction has shown a $0.4 \%$ lattice contraction in films grown at 100 mtorr suggesting substantial $\mathrm{N}$ incorporation[10]). To elucidate the effects of ambient gas pressure on ablation plume dynamics, and to correlate these effects with the RHEED observations and Hall effect measurements, ion probe measurements were performed at several $\mathrm{N}_{2}$ pressures.

Ion current measurements under growth conditions are shown in Figure 2. The figure shows that for some pressure/distance combinations, distinct ion flux distributions or modes are resolvable (e.g., 25 mtorr $\mathrm{N}_{2}$ and 50 mtorr $\mathrm{N}_{2}$ ). These distributions may play a key role in determining the structural and electrical properties of laser ablated films at particular pressure/distance combinations by indicating changes in the dominant growth specie impinging on the substrate. To better illustrate these distributional changes, the ion probe data gathered at each pressure was deconvolved into its constituent ion flux distributions or modes. Depending on the pressure/distance combination, either two or three exponentially-modified-gaussian (EMG) distributions were required to fit each data set. Three distinct EMG distributions were extracted from the entire data set, each having a distinct amplitude and modal velocity (or kinetic energy) which are both functions of pressure and distance. As shown in Figure 3, the distributions are in general agreement with a simple scattering model utilized earlier for ablation of YBCO[24-27], the effective scattering cross-sections in this case ranging from $0.45 \times 10^{-16}$ to $5.1 \times 10^{-16} \mathrm{~cm}^{2}$ as indicated. The boundaries of the shaded region from $\sim 15$ to $\sim 70 \mathrm{mtorr}$ indicate the crossover pressures from mode 1 to mode 2 dominance and from mode 2 to mode 3 dominance, respectively. It is interesting to note that both the Hall mobility and hole concentration maxima lie in this crossover pressure regime. From this observation it is reasonable to conclude that films of the highest quality are achieved when mode 2 is dominant.

Calculations show that at low $\mathrm{N}_{2}$ pressures, including vacuum, the kinetic energy of ablated atoms and ions may be sufficient to produce lattice displacement damage to the growing film's crystalline structure, thereby introducing point defects that reduce both the hole mobility and hole concentration via compensation. In the case of high vacuum the velocity distribution associated 
with mode 1 suggests that at least $75 \%$ of the species have average velocities in the range $0.45 \times 10^{6}$ to $2.313 \times 10^{6} \mathrm{~cm} / \mathrm{s}$, and that $50 \%$ of the species have average velocities $\geq 0.75 \times 10^{6} \mathrm{~cm} / \mathrm{s}$. Spectroscopic inspection of the ablation plume under these conditions has revealed that mode 1 is nearly all atomic. Thus, $\mathrm{Zn}(\mathrm{Te})$, traveling at such velocities would have kinetic energies of $\sim 7$ $181 \mathrm{eV}(\sim 13-354 \mathrm{eV})$ and $19 \mathrm{eV}(37 \mathrm{eV})$, respectively, more than enough to produce lattice damage. Such damage can be mitigated by increasing the $\mathrm{N}_{2}$ pressure in the growth chamber, thereby promoting collisional slowing of the plume and rapid extinction of mode 1 and to a lesser extent, modes 2 and 3 (see Figure 3). By comparing Figures 1 and 3, the crossover from mode 1 to mode 2 is seen to be consistent with an increase in both hole mobility and carrier concentration. Therefore, it is reasonable to conclude that a commensurate reduction in compensation occurs as mode 1 diminishes and as mode 2 begins to dominates. Such improvements in electrical properties with increasing $\mathrm{N}_{2}$ pressure are not without limits, however (see Figure 1). We attribute the reduction in both hole mobility and carrier concentration at $\mathrm{N}_{2}$ pressures in excess of 50 mtorr primarily to excessive $\mathrm{N}$ incorporation and/or growth mode changes revealed by quantitative crosssectional TEM studies.

In this work, defect densities were quantified by preparing cross-sectional TEM specimens for each sample in the $\mathrm{N}_{2}$ pressure series (vacuum, 25, 50, 100 mtorr). Conventional ion milling was used to prepare the specimens and they were analyzed using a JEOL 4000EX operated at 400 $\mathrm{keV}$. Our defect density data is summarized in Figure 4. Aside from the heavily dislocated region near the $\mathrm{ZnTe} / \mathrm{GaAs}$ heterointerface, resulting from the nearly $8 \%$ lattice-mismatch at room temperature, microtwins (as evidenced by selected area diffraction patterns) were the dominant defect in our samples (excluding those displaying a columnar grain structure). Microtwin areal densities were obtained for each sample (again, excluding those displaying a columnar grain structure) by counting the total area of microtwins appearing in a particular volume of $\mathrm{ZnTe}$ above the $\mathrm{ZnTe} / \mathrm{GaAs}$ heterointerface, giving rise to units of $\mathrm{cm}^{2} / \mathrm{cm}^{3}$. This method of counting reflects both the number of defects as well as their extent in the epilayer, and usually presents a more clear picture of defect evolution than a method that involves the number of defects per unit length, for example. It should be noted that the thickness of each cross-sectional sample (i.e., the thickness of the wedge) was not determined as this parameter cancels in the above calculation because it is a factor in both the volume under consideration as well as the twin boundary area. (This assumes that the twin plane extends through the entire wedge, which is an appropriate assumption due to the thinness of the wedge).

The defect density data shown in Figure 4 graphically illustrate the changing microstructure of $\mathrm{ZnTe}$ when deposited at various $\mathrm{N}_{2}$ pressures. The extended defect density increased with increasing nitrogen pressure to 50 mtorr. However, growth at 100 mtorr resulted in a film possessing a columnar grain structure with grain diameters ranging from $\sim 950$ to $1400 \AA$. It was further observed that both the surface of this film as well as near-surface intergranular regions were decorated with spherical particles having diameters ranging from $\sim 95$ to $170 \AA$. We have yet to analyze films deposited in ambients between 50 and 100 mtorr so the precise location of the transition between growth modes is not yet established. The ion probe data, however, offers compelling evidence that the boundary is near 70 mtorr as this is where mode 3 begins to dominate. Irrespective of the exact location of the boundary, the correlation between the ion probe data and the structural data is sufficiently strong to suggest that films grown under mode 3 dominance are products of cluster deposition. The particle distribution and the columnar structure, in particular, appear to result from cluster deposition. Lastly, the low velocity of mode 3 and the size of the decorating particles seem to be consistent with more massive clusters.

\section{CONCLUSIONS}

In summary, we have systematically studied the effect of $\mathrm{N}_{2}$ gas pressure on $\mathrm{ZnTe:N/GaAs}$ film growth and have found that the electrical and microstructural properties of such films are related strongly to gas-controlled variations in the dynamics of the ablation plume. Ion probe measurements, which monitored the attenuation and slowing of the ion current arriving at the substrate surface, indicated that for increasing nitrogen pressure, the fast (vacuum) velocitydistribution splits into a distinct fast and two collisionally-slowed modes. Three distinct pressure regimes were found to exist in which each mode dominated the ion flux impinging on the substrate. Mode 1, dominant below $\sim 15$ mtorr, resulted in a film with the least number of 


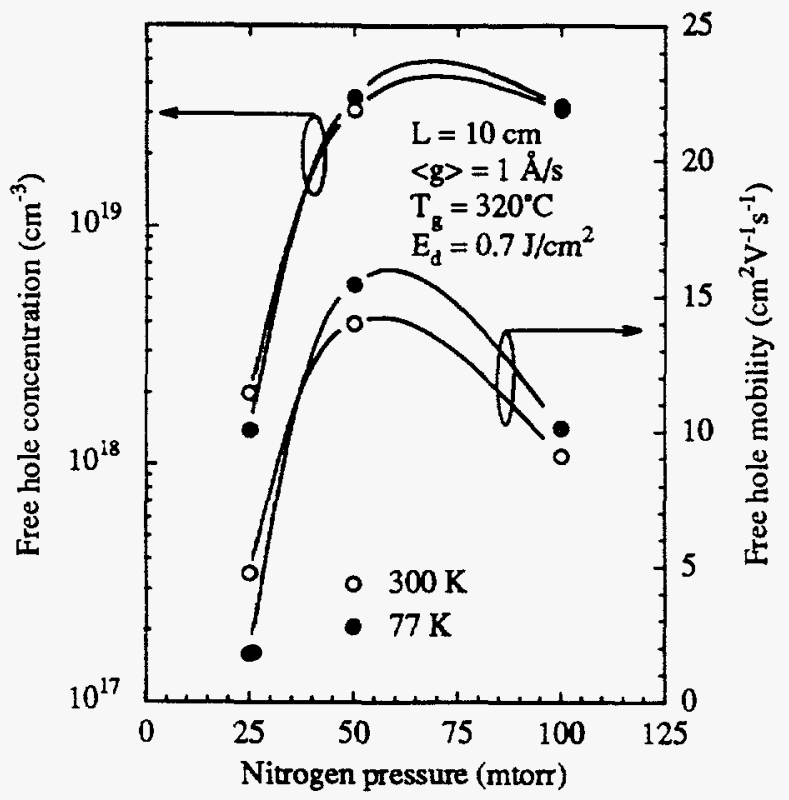

Figure 1. Free hole concentration and mobility in $\mathrm{ZnTe:N/GaAs} \mathrm{heteroepitaxial} \mathrm{films} \mathrm{as} \mathrm{function} \mathrm{of} \mathrm{ambient}$ $\mathrm{N}_{2}$ pressure. Ablation was performed at $10 \mathrm{~cm}$ separation.

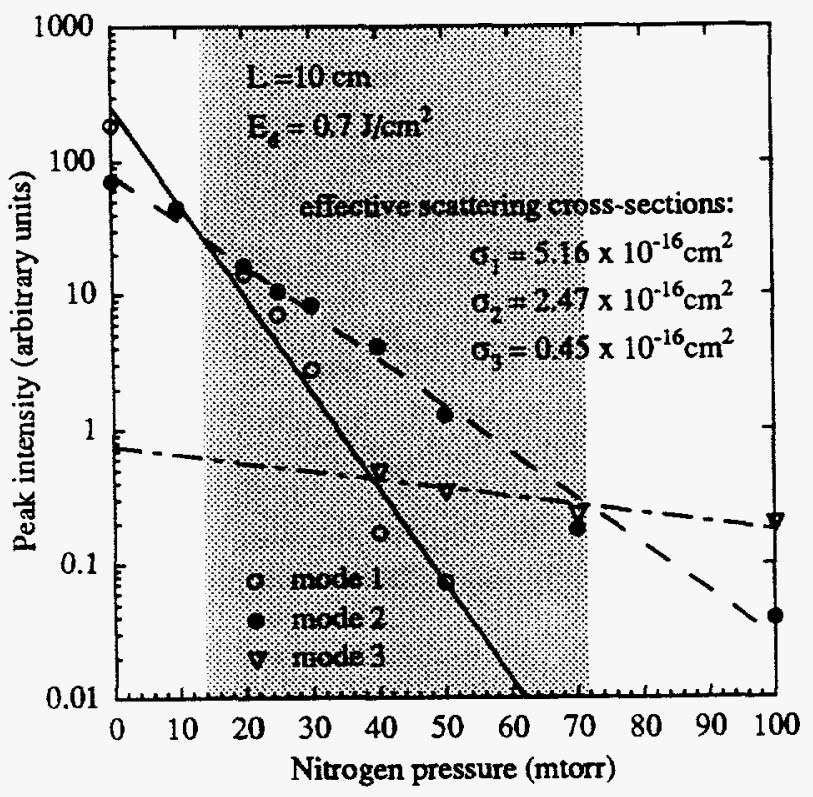

Figure 3. Mode peak intensity as a function of ambient $\mathbf{N}_{2}$ pressure. Ion probe current waveforms measured at several $\mathrm{N}_{2}$ pressures (including those in Figure 2) were deconvolved into distinct modes using exponentiallymodified-gaussian (EMG) distributions. The intensity of these EMG modes were found to decay exponentially with increasing $N_{1}$ pressure and separation according to a simple scattering model (see text).

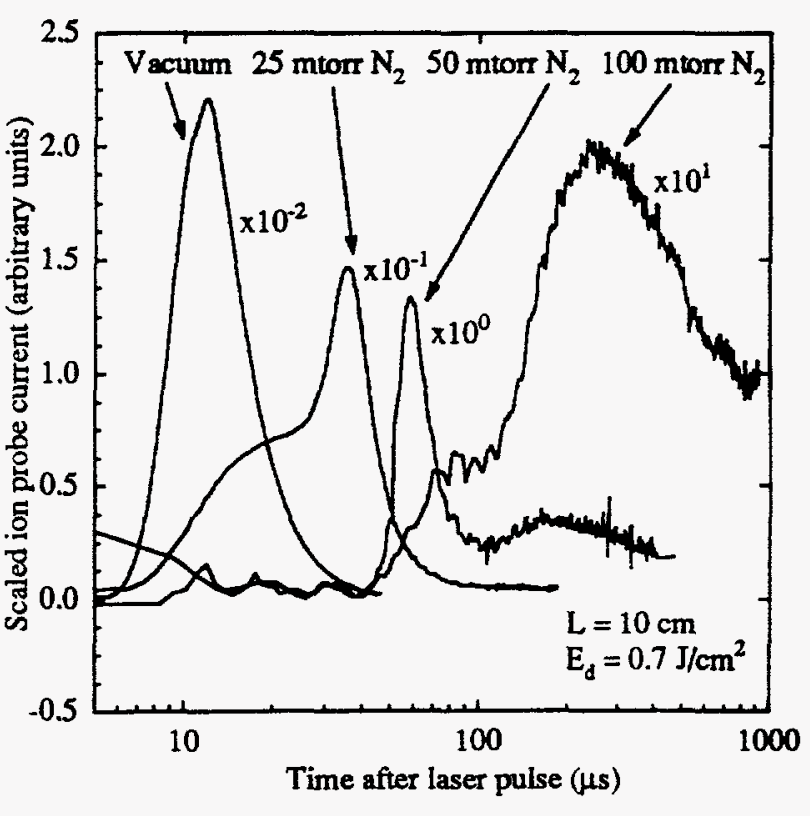

Figure 2. Ion probe current waveforms measured $10 \mathrm{~cm}$ from the ablation target as a function of ambient $\mathrm{N}_{2}$ pressure. Note the relative scaling of each waveform and the appearance of distinct modes at 25 and 50 mtorr.

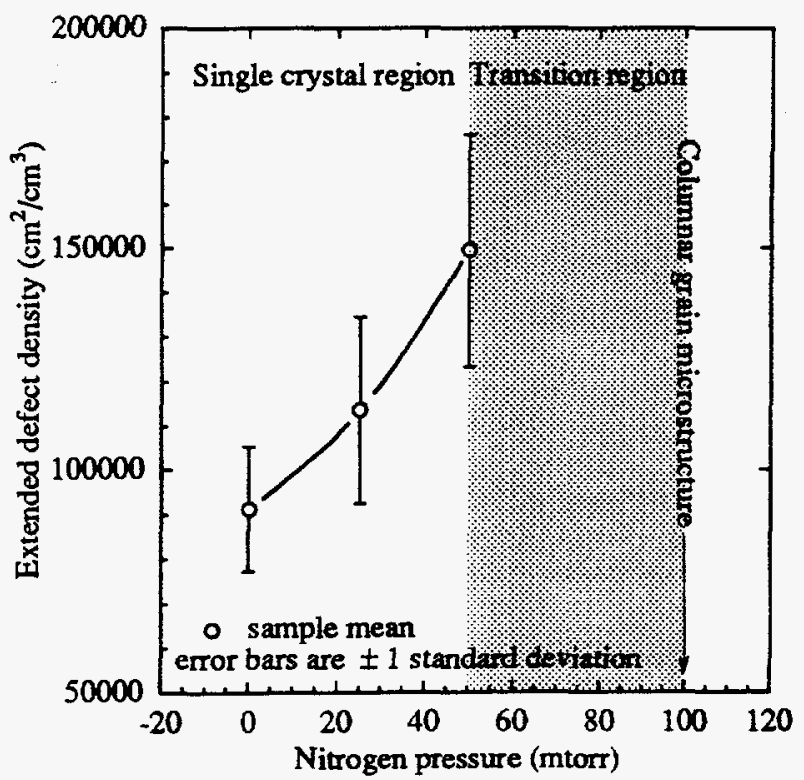

Figure 4. Extended defect density in laser ablated $\mathrm{ZnTe} / \mathrm{GaAs}$ films as a function of ambient $\mathrm{N}_{2}$ pressure. Note the transition from a single crystal microstructure to that of columnar grains.

extended defects although it is not clear at this time whether films can be grown in this regime with optimum optoelectronic properties. Mode 2, dominant from $\sim 15$ to $\sim 70$ mtorr, seems to be necessary for high quality doped films as evidenced by a high carrier concentration and hole mobility. Lastly, mode 3, which dominated the ion flux after $\sim 70 \mathrm{mtorr}$, is consistent with the onset of a cluster deposition regime, as suggested by cross-sectional TEM studies showing a distinctive change in film microstructure. 


\section{ACKNOWLEDGMENTS}

The authors gratefully acknowledge P.H. Fleming for preparing the Hall effect samples. This work was sponsored by the Division of Materiais Science, U.S. Department of Energy under contract DE-AC05-840R21400 with Lockheed-Martin Energy Systems, Inc. and supported in part by an appointment to the Oak Ridge National Laboratory Postdoctoral Research Associates Program administered jointly by the Oak Ridge National Laboratory and the Oak Ridge Institute for Science and Education.

\section{REFERENCES}

1. D. Roy and S.B. Krupanidhi, J. Mater. Res. 7, 2521 (1992).

2. H.S. Kim and H.S. Kwok, Appl. Phys. Lett. 61, 2234 (1992).

3. R. P. Casero, F. Kerherve, J.P. Enard and J. Perriere, Applied Surface Science 54, 147 (1992).

4. M.C. Foote, B.B. Jones, B.D. Hunt, J.B. Bamer, R.P. Vasquez and L.J. Bajuk, Physica C 201, 176 (1992).

5. J. Lee, E. Narumi, C. Li, S. Patel and D.T. Shaw, Physica C 200, 235 (1992).

6. W.P Shen and H.S. Kwok, in Compound Semiconductor Epitaxy MRS symposium proceedings V340, edited by C.W. Tu, L.A. Kolodziejski and V.R. McCrary, (MRS, Pittsburgh, PA, 1994), p 457.

7. J.W. McCamy and D.H. Lowndes, Appl. Phys. Lett. 63, 3008 (1993).

8. M.Y. Chem, H.M. Lin, C.C. Fang, J.C. Fan and Y.F. Chen, Appl. Phys. Lett. 67, 1390 (1995).

9. W.P. Shen and H.S. Kwok, Appl. Phys. Lett. 65, 2162 (1994).

10. C.M. Rouleau, D.H. Lowndes, J.W. McCamy. J.D. Budai, D.B. Poker, D.B. Geohegan, A.A. Puretzky and S. Zhu, Appl. Phys. Lett. 67, 2545 (1995).

11. W.P. Shen and H.S. Kwok, in Film Synthesis and Growth Using Energetic Beams MRS symposium proceedings V388, edited by H.A. Atwater, J.T. Dickinson, D.H. Lowndes and A. Polman, (MRS, Pittsburgh, PA 1995), p 91.

12. D.H. Lowndes, C.M. Rouleau, J.W. McCamy, J.D. Budai, D.B. Poker, D.B. Geohegan, A.A. Puretzky and S. Zhu, in Film Synthesis and Growth Using Energetic Beams MRS symposium proceedings V388, edited by H.A. Atwater, J.T. Dickinson, D.H. Lowndes and A. Polman, (MRS, Pittsburgh, PA, 1995), p. 85.

13. H. Uchiki, O. Machida, A. Tanaka and H. Hirasawa, Jpn. J. Appl. Phys. 32, L764 (1993).

14. E. Ahmed, A.E. Hill, J. Leppavuori, R.D. Pilkington, R.D. Tomlinson, J. Levoska and 0. Kusmartseva, Adv. Materials for Optics and Elect. 4, 423 (1994).

15. R. Schaffler, M. Klose, M. Brieger, H. Dittrich and H.W. Schock, Materials Sci. Forum 173174, 135 (1995).

16. J. Levoska, A.E. Hill, S. Leppavuori, O. Kusmartseva, R.D. Tomlinson and R.D. Pilkington, Jpn J. Appl. Phys. 32, 43 (193).

17. V.F. Gremenok, E.P. Zaretskava, I.V. Bodnar and I.A. Victorov, Jpn. J. Appl. Phys. 32, 90 (1993).

18. J. Levoska, S. Leppavuori, F. Wang, O. Kusmartseva, A.E. Hill, E. Ahmed, R.D. Tomlinson and R.D. Pilkington, Physica Scripta T54, 244 (1994).

19. A.E. Hill, S. Leppavuori, R.D. Tomlinson, R.D. Pilkington, J. Levoska, E. Ahmed, J. Franti, in Laser Ablation in Materials Processing: Fundamentals and Applications MRS symposium proceedings V285, edited by B. Braren, J.J. Dubowski and D.P. Norton, (MRS, Pittsburgh, PA 1993), p. 483.

20. H. Uchiki, H. Hirasawa and I. Hasegawa, Jpn. J. Appl. Phys. 33, L983 (1994).

21. D. Feiler and R. S. Williams, Appl. Phys. Lett., submitted for publication.

22. D. Feiler, R.S. Williams, A.A. Talin, H. Yoon and M.S. Goorsky, J. Cryst. Growth, submitted for publication.

23. D.H. Lowndes, C.M. Rouleau, D.B. Geohegan, A.A. Puretzky, M.A. Strauss, A.J. Pedraza, J.W. Park, J.D. Budai and D.B. Poker, Pulsed Laser Ablation Growth and Doping of Epitaxial Compound Semiconductor Films, these proceedings.

24. D.B. Geohegan, in Laser Ablation: Mechanisms and Applications, edited by J.C. Miller and R.F. Haglund, (Springer-Verlag, Heodelberg, 1991), p. 28.

25. D.B. Geohegan, in Pulsed Laser Deposition of Thin Films, edited by D.B. Chrisley and G.K. Hubler, (Wiley, New York, 1994), chap 5.

26. D.B. Geohegan, Thin Solid Films 220, 138 (1992).

27. D.B. Geohegan, in Laser Ablation of Electronic Materials: Basic Mechanisms and Applications, edited by E. Fogarassy and S. Lazare, (North Holland, Amsterdam, 1992), p. 73. 